\title{
Homogenization of a reaction-diffusion system modeling sulfate corrosion of concrete in locally periodic perforated domains
}

\author{
Tasnim Fatima • Nasrin Arab • \\ Evgeny P. Zemskov • Adrian Muntean
}

Received: 21 August 2009 / Accepted: 12 July 2010 / Published online: 31 July 2010

(C) The Author(s) 2010. This article is published with open access at Springerlink.com

\begin{abstract}
A reaction-diffusion system modeling concrete corrosion in sewer pipes is discussed. The system is coupled, semi-linear, and partially dissipative. It is defined on a locally periodic perforated domain with nonlinear Robin-type boundary conditions at water-air and solid-water interfaces. Asymptotic homogenization techniques are applied to obtain upscaled reaction-diffusion models together with explicit formulae for the effective transport and reaction coefficients. It is shown that the averaged system contains additional terms appearing due to the deviation of the assumed geometry from a purely periodic distribution of perforations for two relevant parameter regimes: (a) all diffusion coefficients are of order of $\mathcal{O}(1)$ and (b) all diffusion coefficients are of order of $\mathcal{O}\left(\varepsilon^{2}\right)$ except the one for $\mathrm{H}_{2} \mathrm{~S}(\mathrm{~g})$ which is of order of $\mathcal{O}(1)$. In case $(a)$ a set of macroscopic equations is obtained, while in case $(b)$ a two-scale reaction-diffusion system is derived that captures the interplay between microstructural reaction effects and the macroscopic transport.
\end{abstract}

Keywords Asymptotic homogenization · Locally periodic perforated media · Nonlinear Robin-type boundary conditions $\cdot$ Semi-linear PDE-ODE system $\cdot$ Sulfate corrosion

Nasrin Arab-Visiting during spring 2009 CASA, Department of Mathematics and Computer Science, Technical University Eindhoven, The Netherlands.

T. Fatima $(\varangle) \cdot$ A. Muntean

CASA - Centre for Analysis, Scientific Computing and Applications, Department of Mathematics and Computer Science, Institute for Complex Molecular Systems (ICMS), Technical University Eindhoven, Eindhoven, The Netherlands e-mail: t.fatima@tue.nl

A. Muntean

e-mail: a.muntean@tue.nl

N. Arab

De Lismortel 246, 5612 AK Eindhoven, The Netherlands

e-mail: nasrin.arab@gmail.com

E. P. Zemskov

Continuum Mechanics Department, Computing Centre of the Russian Academy of Sciences, Moscow, Russia

e-mail: zemskov@ccas.ru 


\section{Abbreviations \\ PDE Partial differential equation \\ ODE Ordinary differential equation \\ RD Reaction diffusion}

\section{Introduction}

Sulfuric acid is the cause of severe attack to concrete in sewerage systems. Although normally sewage does not affect the concrete matrix, under some conditions (like raised local temperature activating anaerobic bacteria of the species Desulfovibrio desulfuricans, e.g., and a suitable $\mathrm{pH}$ range) considerable production of hydrogen sulfide $\mathrm{H}_{2} \mathrm{~S}$ takes place and leads to acid attack [1]. This situation can be briefly described as follows: $\mathrm{H}_{2} \mathrm{~S}$ present in the air space of a sewer dissolves in stationary moisture films on the exposed concrete surfaces where it undergoes oxidation by aerobic bacteria to sulfuric acid. The chemical attack seems to take place only on the roof and upper part of the sewer where it finally leads to damage, i.e., spalling of the material.

In spite of the fact that concrete has a long satisfactory service in sewarage systems, no hydraulic cement can withstand the acidity caused by the anaerobic conditions. In this paper, we focus our attention on forecasting the early stage of the corrosion. ${ }^{1}$

We consider a semilinear reaction-diffusion system which we refer to as micro-model, see Sect. 2.3 for the details. This describes the evolution of gaseous and dissolved $\mathrm{H}_{2} \mathrm{~S}$, as well as of the sulfuric acid $\mathrm{H}_{2} \mathrm{SO}_{4}$, moisture, and gypsum at the pore level. Having as departure point a micro-model for this reaction-diffusion (RD) scenario, we want to derive, by means of asymptotic homogenization techniques, macroscopic RD models able to describe accurately the initiation of sulfate corrosion in sewer pipes. As a further step, the "homogenized" models need to be tested against experimental findings at the macroscopic level and calibrated in order to forecast the penetration of the acid front.

A few basic questions are relevant at this stage:

(i) What would be "reasonable" assumptions that we may make concerning the microstructure of the concrete pipe? How much freedom do we have for a deterministic averaging strategy?

(ii) Does the resulting macro-model approximate well the rather complex multi-scale physico-chemical situation?

(iii) How good is/can be this approximation?

Since the analysis we report here is only preliminary, we address particularly question (i) and leave questions (ii) and (iii) unanswered for the moment.

The paper is organized as follows: In Sect. 2 we give a minimal modeling at the pore level of the relevant physico-chemical processes involved in the early stage of sulfate corrosion of cement-based materials and explain both the flexibility and limitations of our modeling. We define in Sect. 2.1 a periodic-cells approximation of the part of the concrete pipe we are looking at, as well as the corresponding locally periodic array of perforations. We nondimensionalize in Sect. 3 the micro-model presented in Sect. 2.3. The homogenization procedure, the macro and micro-macro mass-balance equations, together with a list of effective transport and reaction coefficients are presented in Sect. 4.

\subsection{A few comments on related literature}

The reader can find details on civil-engineering aspects concerning concrete-corrosion issues when acid attack is involved, for instance, in [2-7]. We particularly like [2] for the clear exposition of the phenomenology and for the

\footnotetext{
1 Whitish surface deposits appear, but the mechanical properties of the material stay unaffected. Note that, at a later stage, a gradual softening of the cement paste appears and mechanical destabilization of the microstructure takes place. The literature reports rates of corrosion of about $6-12 \mathrm{~mm}$ penetration depth per year.
} 
enumeration of the main mechanisms influencing acid corrosion. A standard reference work concerning cement chemistry is [8].

From the modeling point of view, we were very much inpired by [9] (see also the subsequent papers [10,11]), where the authors adopted a macroscopic moving-boundary modeling strategy to capture the macroscopic corrosion front penetrating the pipe. We adapted some of their modeling ideas for the micro-model proposed in Sect. 2.3. Another macroscopic approach for a closely related sulfatation problem has been reported in [12]. In this paper, we keep a reduced size of the micro-model. The reader can easily extend this model to allow for more complex chemistry (like coupling sulfatation with carbonation reactions) or to include a more detailed modeling of the flow (by considering the evolution of both water vapors and liquid water).

At the technical level, we essentially use formal asymptotics techniques for both the periodic and locally periodic homogenization. We refer the reader to [13] for a discussion on uniform descriptions of heterogeneous media, while the working technique is detailed for instance in [14, pp. 11-22], [15, pp. 2-10], and [16, Chap. 7]; see also [17] for a related application. The references [16], [18-20] contain more theoretical approaches able to justify the asymptotics at least for simpler PDE models.

Homogenization problems in locally periodic perforated domains have been dealt with in, for instance, [18], [21-23]; see [24] for a more recent account of bibliographic information. At the technical level, we rely on the analysis reported in [23] for the case of a Poisson problem with a linear Fourier condition imposed at the boundary of the perforation. We assume a locally periodic distribution of the perforations (i.e., of the micropores). By this we step away from the often-used periodic approximation of porous media, which for the particular case of concrete is much too rough. Moreover, we expect that some randomness is needed for better covering of what happens in reality, but we prefer for the moment to stick with a deterministic approach and understand [for this easier case] the occurrence of new terms expressing deviations from periodicity.

Structured transport in porous media, like that arising when gaseous and dissolved chemical species (here: $\mathrm{H}_{2} \mathrm{~S}(g)$ and $\mathrm{H}_{2} \mathrm{~S}(a q)$ ) diffuse simultaneously, multi-spatial-scale situations naturally occur [25-28]. Many of these models can be derived rigorously by means of homogenization techniques [29]. Note that the formal analysis done for a two-scale setting in Sect. 3.3.2 of [26] remotely ressembles ours for the case (2).

\section{Modeling sulfate corrosion in sewer pipes}

In this section, we describe the geometry of a typical sewer system and present our concept of microstructure. Next we recall the physical and chemical mechanisms that we take into account, and finally, we list the equations entering our micro-model.

\subsection{Description of the problem and geometry}

We consider a cross-section of a sewerage pipe made of partially wet concrete. It is worth noting that concrete is a mixture of cement, gel and mobile water as well as of aggregate (sand, gravel, etc). Therefore we assume that any microstructure (any representative cell) contains three non-overlapping regions: the solid matrix (aggregate, eventually inaccessible-to-diffusion gel water, cement paste, etc.), the pore water clinging on solid fabrics as well as the air-filled part of the pore; see Fig. 1 for a sketch of the cell geometry, say $Y$, divided into three (distinct and non-mixed) components: solid, water, and air. We assume that the solid part is placed in the center of the cell which is enclosed by a stationary water film. Around the water film, we assume the presence of bulk air as shown in Fig. 1 (bottom). Additionally, we assume that the domain of interest can be approximated by a finite union of this kind of cells.

Let us now have a look at our perforations: each cell contains two internal interfaces: one separating the solid part from the water film, and the second separating the water film from the air part. We consider the following constraints to be fulfilled: 


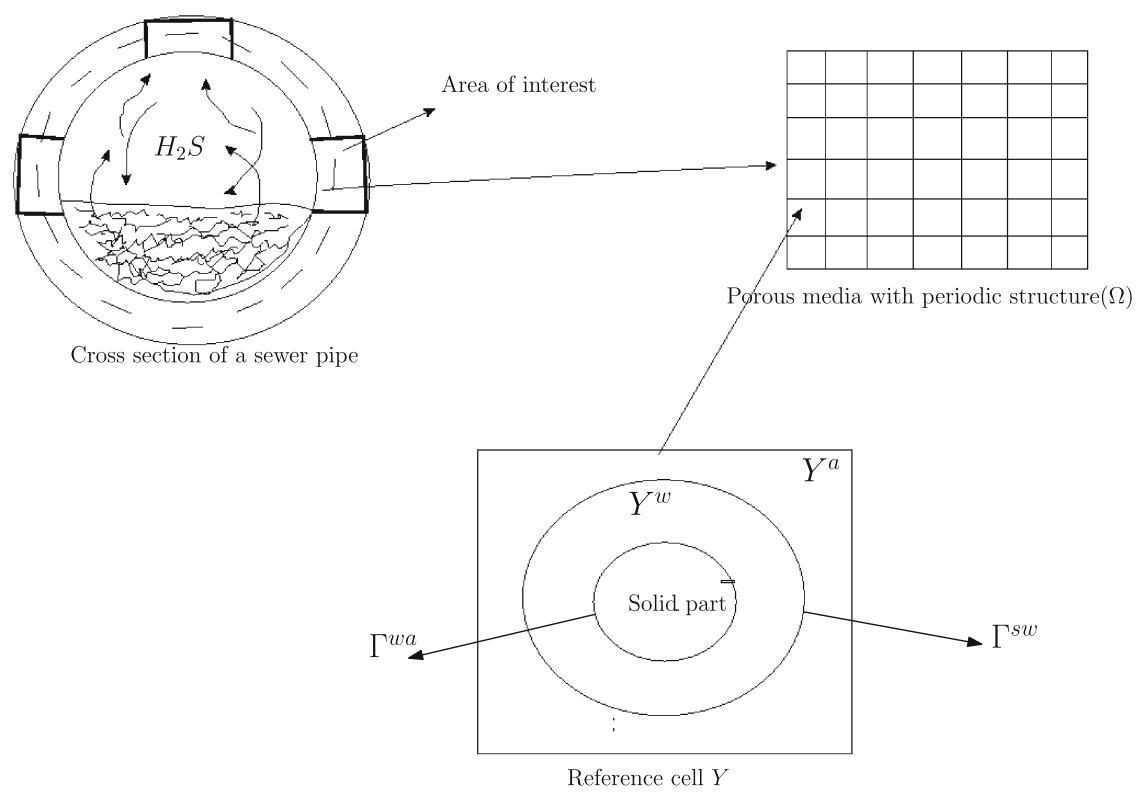

Fig. 1 Top left: cross-section of a sewer showing 3 critical regions where corrosion initiates; Top right: periodic grid covering one of the critical regions; Bottom: typical pore/reference cell

(i) each cell contains all three regions: solid, air, and water. None of them disappears during the RD process. The shapes of their outer boundaries do not evolve with time, ${ }^{2}$ but are allowed to be $x$-dependent. This means that they may be different at different space positions.

(ii) the $x$-dependency of the internal interfaces mentioned in (i) is locally periodic.

(iii) all internal interfaces are sufficiently smooth and do not touch each other.

Assumptions (i), (ii), (iii) should not be seen as very restrictive. They do cover many physically interesting situations.

Usually, in periodic homogenization approaches (like in $[14,16])$ the shape of these interfaces (i.e., the boundary of the perforations) and is $x$-independent. If the shape of the internal interfaces in the cell is not $x$-dependent, then the outer normals to these interfaces depend on the fast variable $y=x / \epsilon$ only. Hence, oscillations of the internal boundaries from cell to cell can no longer be captured. As a direct consequence, macroscopic factors like porosity or air- and water-fractions, are positive numbers. ${ }^{3}$

We will notice in Sect. 4 that the dependence of the normal vectors to the active internal interfaces on both the $x$ - and $y$-variables involves difficulties at the technical level, but the fact that (ii) holds will be very helpful in controlling (at least formally) the oscillations.

\subsection{Notation}

Let $\Omega$ be an open set in $\mathbb{R}^{3}$ with a smooth boundary $\Gamma$ having two disjoint pieces $\Gamma^{D}$ and $\Gamma^{N}$. Here $\Gamma^{D} \cup \Gamma^{N}=\Gamma$ and $\mu\left(\Gamma^{D}\right) \neq 0$, where $\mu$ is the (surface) Lebesque measure in $\mathbb{R}^{2}$. The domain $Y$ is the reference cell in $\mathbb{R}^{3}$, while $S:=(0, T)$ is the time interval; $Y$ splits up into $Y^{a}$ which denotes the air-filled part of the cell, $Y^{w}$ is the water-filled part of the cell, and $Y^{s}$ the solid part of the cell. Furthermore,

${ }^{2}$ Reference [30] reports on a homogenization procedure which can deal (unfortunately only) formally with evolving microstructures for a precipitation/dissolution problem.

3 In particular, they do not depend on the $x$-variable. 
$Y:=Y^{w} \cup Y^{s} \cup Y^{a}$ with $Y^{w} \cap Y^{s} \cap Y^{a}=\emptyset$.

Also, we denote by $\Gamma^{s w}:=\partial Y^{s}$ the interface between water and the solid part of the cell and $\Gamma^{w a}:=\partial Y^{w}$ is the interface between the water-filled and air-filled part of the cell. By $\Theta_{x}$ we indicate that the micro-domain $\Theta \subset \Omega$ depends explicitly on the macroscopic space position $x$, while by $\Theta_{\varepsilon}$ we point out that the micro-domain $\Theta$ oscillates when changing the resolution $\varepsilon>0$.

\subsubsection{Periodic array of perforations}

For a subset $X$ of $Y$ and the integer vectors $k=\left(k_{1}, k_{2}, k_{3}\right) \in \mathbb{Z}^{3}$, we denote the shifted subset by

$X_{k}:=X+\sum_{i=1}^{3} k_{i} e_{i}$,

where $e_{i}$ is the $i$ th unit vector in $\mathbb{R}^{3}$.

We assume that $\hat{\Omega}_{\varepsilon}$ is made up of copies of the unit cell scaled by a sufficiently small scaling factor $\varepsilon>0$. Here $\varepsilon$ is a small parameter whose precise meaning will become clear in Sect. 3.

Further:

$\hat{\Omega}_{\varepsilon}:=\hat{Y}_{\varepsilon}^{a} \cup \hat{Y}_{\varepsilon}^{w} \cup \hat{Y}_{\varepsilon}^{s}$;

$\hat{Y}_{\varepsilon}^{a}:=\bigcup_{k \in \mathbb{Z}^{3}}\left\{\varepsilon Y_{k}^{a} \mid \varepsilon Y_{k}^{a} \subset \Omega\right\}$, the air-filled part of the pores;

$\hat{Y}_{\varepsilon}^{w}:=\bigcup_{k \in \mathbb{Z}^{3}}\left\{\varepsilon Y_{k}^{w} \mid \varepsilon Y_{k}^{w} \subset \Omega\right\}$, the water-filled part of the pores;

$\hat{Y}_{\varepsilon}^{s}:=\bigcup_{k \in \mathbb{Z}^{3}}\left\{\varepsilon Y_{k}^{s} \mid \varepsilon Y_{k}^{s} \subset \Omega\right\}$, solid matrix;

$\hat{\Gamma}_{\varepsilon}^{s w}:=\bigcup_{k \varepsilon Z^{n}}\left\{\varepsilon \Gamma_{k}^{s w} \mid \varepsilon \Gamma_{k}^{s w} \subset \Omega\right\}$ water-solid interface;

$\hat{\Gamma}_{\varepsilon}^{w a}:=\bigcup_{k \varepsilon Z^{n}}\left\{\varepsilon \Gamma_{k}^{w a} \mid \varepsilon \Gamma_{k}^{w a} \subset \Omega\right\}$ water-air interface.

\subsubsection{Locally periodic array of perforations}

In the locally periodic setting, one represents the normal vector $n^{\varepsilon}$ to the "oscillating" internal boundaries of the perforations in the form suggested, for instance, in $[18,22]$ as follows:

$n^{\varepsilon}(x, y):=\tilde{n}(x, y)+\varepsilon n^{\prime}(x, y)+\mathcal{O}\left(\varepsilon^{2}\right)$,

where

$\tilde{n}(x, y):=\frac{\nabla_{y} P(x, y)}{\left|\nabla_{y} P(x, y)\right|}$

and

$n^{\prime}(x, y):=\frac{\nabla_{x} P(x, y)}{\left|\nabla_{y} P(x, y)\right|}-\nabla_{y} P(x, y) \frac{\nabla_{x} P(x, y), \nabla_{y} P(x, y)}{\left|\nabla_{y} P(x, y)\right|^{3}}$.

Here the generic surface $P(x, y)$, which describes the interfaces ${ }^{4} \Gamma_{\varepsilon}^{s w}, \Gamma_{\varepsilon}^{w a}$, and $\Gamma_{\varepsilon}$, respectively, is assumed to be a 1-periodic function in the variable y and sufficiently smooth with respect to both variables $x, y$. These functions $P(x, \cdot)$ are assumed to be given for each $x \in \Omega$.

$\overline{4} \Gamma_{\varepsilon}^{s w}, \Gamma_{\varepsilon}^{w a}$, and $\Gamma_{\varepsilon}$ point out the same class of objects as those defined in the periodic setting with the same name under a hat, but now the periodicity assumption is removed. The same statement holds for $\Omega_{\varepsilon}, Y_{\varepsilon}^{a}, Y_{\varepsilon}^{w}$, and $Y_{\varepsilon}^{s}$. Note that this notation hides the existing dependence of the geometry on the parameter $x$. 


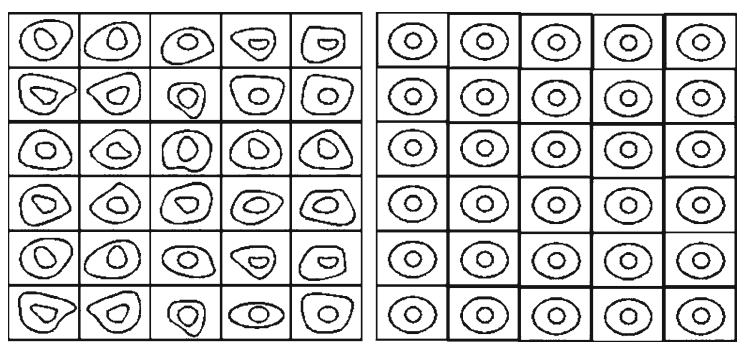

Fig. 2 Left: locally periodic array of perforations. Right: uniformly periodic array of perforations. In the two pictures, we expect the occurrence of differences at most of the order of $\mathcal{O}(\varepsilon)$ between any two corresponding inner interfaces

It is worth noting that, for uniformly periodic perforations, $\tilde{n}$ only depends on $y$ and $n^{\prime}=0$. To give meaning to the formal calculations that we perform in this paper, it suffices to define the local periodicity appearing in the geometry from 2 (left) using the description (2) of the normal vectors to the non-periodically placed interfaces.

We refer the reader to [22] for an accurate mathematical description of the geometry described in Fig. 2 (left) and to [21] for connections between locally periodic perforated domains and quasi-periodic functions. See [19] for a notation strategy for the periodic case.

\subsection{Micro-model}

List of data and unknowns

The data is given by:

$u_{10}: \Omega \longrightarrow \mathbb{R}_{+}$一the initial concentration of $\mathrm{H}_{2} \mathrm{SO}_{4}(a q)$

$u_{20}: \Omega \longrightarrow \mathbb{R}_{+}$- the initial concentration of $\mathrm{H}_{2} \mathrm{~S}(a q)$

$u_{30}: \Omega \longrightarrow \mathbb{R}_{+}$一 the initial concentration of $\mathrm{H}_{2} \mathrm{~S}(\mathrm{~g})$

$u_{40}: \Omega \longrightarrow \mathbb{R}_{+}$- the initial concentration of moisture

$u_{50}: \Omega \longrightarrow \mathbb{R}_{+}$- the initial concentration of dissolved gypsum

$u_{3}^{D}: \Gamma_{D} \times S \longrightarrow \mathbb{R}_{+}$- exterior concentration (Dirichlet data) of $\mathrm{H}_{2} \mathrm{~S}(\mathrm{~g})$.

The unknowns are:

$u_{1}^{\varepsilon}: Y_{\varepsilon}^{w} \times S \longrightarrow \mathbb{R}$-mass concentration of $\mathrm{H}_{2} \mathrm{SO}_{4}(a q)\left[\mathrm{g} / \mathrm{cm}^{3}\right]$

$u_{2}^{\varepsilon}: Y_{\varepsilon}^{w} \times S \longrightarrow \mathbb{R}$-mass concentration of $\mathrm{H}_{2} \mathrm{~S}(a q)\left[\mathrm{g} / \mathrm{cm}^{3}\right]$

$u_{3}^{\varepsilon}: Y_{\varepsilon}^{a} \times S \longrightarrow \mathbb{R}$-mass concentration of $\mathrm{H}_{2} \mathrm{~S}(\mathrm{~g})\left[\mathrm{g} / \mathrm{cm}^{3}\right]$

$u_{4}^{\varepsilon}: Y_{\varepsilon}^{w} \times S \longrightarrow \mathbb{R}$-mass concentration of moisture $\left[\mathrm{g} / \mathrm{cm}^{3}\right]$

$u_{5}^{\varepsilon}: \Gamma_{\varepsilon}^{s w} \times S \longrightarrow \mathbb{R}$-mass concentration of gypsum $\left[\mathrm{g} / \mathrm{cm}^{2}\right]$.

The mass-balance equation for $\mathrm{H}_{2}(\mathrm{SO})_{4}$ is:

$\partial_{t} u_{1}^{\varepsilon}+\operatorname{div}\left(-d_{1}^{\varepsilon} \nabla u_{1}^{\varepsilon}\right)=-k_{1}^{\varepsilon} u_{1}^{\varepsilon}+k_{2}^{\varepsilon} u_{2}^{\varepsilon}, \quad x \in Y_{\varepsilon}^{w}, t \in S$

$u_{1}^{\varepsilon}(x, 0)=u_{10}^{\varepsilon}(x), \quad x \in Y_{\varepsilon}^{w}$

$n^{\varepsilon} \cdot\left(-d_{1}^{\varepsilon} \nabla u_{1}^{\varepsilon}\right)=0, \quad x \in \Gamma_{\varepsilon}^{w a}, t \in S$

$n^{\varepsilon} \cdot\left(-d_{1}^{\varepsilon} \nabla u_{1}^{\varepsilon}\right)=\eta\left(u_{1}^{\varepsilon}, u_{5}^{\varepsilon}\right), \quad x \in \Gamma_{\varepsilon}^{s w}, t \in S$.

The mass-balance equation for $\mathrm{H}_{2} \mathrm{~S}(a q)$ is given by:

$\partial_{t} u_{2}^{\varepsilon}+\operatorname{div}\left(-d_{2}^{\varepsilon} \nabla u_{2}^{\varepsilon}\right)=k_{1}^{\varepsilon} u_{1}^{\varepsilon}-k_{2}^{\varepsilon} u_{2}^{\varepsilon}, \quad x \in Y_{\varepsilon}^{w}, t \in S$

$u_{2}^{\varepsilon}(x, 0)=u_{20}^{\varepsilon}(x), \quad x \in Y_{\varepsilon}^{w}$

$n^{\varepsilon} \cdot\left(-d_{2}^{\varepsilon} \nabla u_{2}^{\varepsilon}\right)=a^{\varepsilon}(x) u_{3}^{\varepsilon}-b^{\varepsilon}(x) u_{2}^{\varepsilon}, \quad x \in \Gamma_{\varepsilon}^{w a}, t \in S$

$n^{\varepsilon} \cdot\left(-d_{2}^{\varepsilon} \nabla u_{2}^{\varepsilon}\right)=0, \quad x \in \Gamma_{\varepsilon}^{s w}, t \in S$. 
The mass-balance equation for $\mathrm{H}_{2} \mathrm{~S}(g)$ is given by:

$\partial_{t} u_{3}^{\varepsilon}+\operatorname{div}\left(-d_{3}^{\varepsilon} \nabla u_{3}^{\varepsilon}\right)=0, \quad x \in Y_{\varepsilon}^{a}, t \in S$

$u_{3}^{\varepsilon}(x, 0)=u_{30}^{\varepsilon}(x), \quad x \in Y_{\varepsilon}^{a}$

$n^{\varepsilon} \cdot\left(-d_{3}^{\varepsilon} \nabla u_{3}^{\varepsilon}\right)=0, \quad x \in \Gamma^{N}, t \in S$

$u_{3}^{\varepsilon}(x, t)=u_{3}^{D}(x, t), \quad x \in \Gamma^{D}, t \in S$

$n^{\varepsilon} \cdot\left(-d_{3}^{\varepsilon} \nabla u_{3}^{\varepsilon}\right)=-\left(a^{\varepsilon}(x) u_{3}^{\varepsilon}-b^{\varepsilon}(x) u_{2}^{\varepsilon}\right), \quad x \in \Gamma_{\varepsilon}^{w a}, t \in S$.

The mass-balance equation for moisture is given by:

$\partial_{t} u_{4}^{\varepsilon}+\operatorname{div}\left(-d_{4}^{\varepsilon} \nabla u_{4}^{\varepsilon}\right)=k_{1}^{\varepsilon} u_{1}^{\varepsilon}, \quad x \in Y_{\varepsilon}^{w}, t \in S$

$u_{4}^{\varepsilon}(x, 0)=u_{40}^{\varepsilon}(x), \quad x \in Y_{\varepsilon}^{w}$

$n^{\varepsilon} \cdot\left(-d_{4}^{\varepsilon} \nabla u_{4}^{\varepsilon}\right)=0, \quad x \in \Gamma_{\varepsilon}^{w a}, t \in S$

$n^{\varepsilon} \cdot\left(-d_{4}^{\varepsilon} \nabla u_{4}^{\varepsilon}\right)=0, \quad x \in \Gamma_{\varepsilon}^{s w}, t \in S$.

The mass-balance equation for the gypsum present at the water-solid interface is:

$\partial_{t} u_{5}^{\varepsilon}=\eta\left(u_{1}^{\varepsilon}, u_{5}^{\varepsilon}\right), \quad x \in \Gamma_{\varepsilon}^{s w}, t \in S$

$u_{5}^{\varepsilon}(x, 0)=u_{50}^{\varepsilon}(x), \quad x \in \Gamma_{\varepsilon}^{s w}$.

Note that the lack of diffusion in (9) lends the partly dissipative feature to the model.

The list of coefficients in (5)-(9) is as follows:

$k_{j}^{\varepsilon}: \Omega \times S \longrightarrow \mathbb{R}$-reaction constants for all $j \in\{1,2,3\}$,

$d_{i}^{\varepsilon}: \Omega \times S \longrightarrow \mathbb{R}^{3 \times 3}$ —diffusion coefficients for $\mathrm{H}_{2} \mathrm{SO}_{4}, \mathrm{H}_{2} \mathrm{~S}(a q), \mathrm{H}_{2} \mathrm{~S}(g)$ and $\mathrm{H}_{2} \mathrm{O}$ for all $i \in\{1,2,3,4\}$,

$a^{\varepsilon}: \Gamma_{\varepsilon}^{w a} \times S \longrightarrow \mathbb{R}$-the adsorption factor of $\mathrm{H}_{2} \mathrm{~S}$ (air to water),

$b^{\varepsilon}: \Gamma_{\varepsilon}^{w a} \times S \longrightarrow \mathbb{R}$-the desorption factor of $\mathrm{H}_{2} \mathrm{~S}$ (air to water),

$\eta: \Gamma_{\varepsilon}^{s w} \times S \longrightarrow \mathbb{R}$-reaction rate on the water-solid interface $\eta: \Gamma_{\varepsilon}^{s w}$.

It is tacitly assumed that all reaction constants, diffusion coefficients, absorption, and desorption factors as well as normal vectors to the water-solid and water-air interfaces are $Y$-periodic functions as follows: $d_{i}^{\varepsilon}(x, t):=$ $d_{i}(x / \varepsilon, t), i \in\{1,2,3,4\}, k_{j}^{\varepsilon}(x, t):=k_{j}(x / \varepsilon, t), j \in\{1,2,3\} ; a^{\varepsilon}(x, t):=a(x / \varepsilon, t)$, and $b^{\varepsilon}(x, t):=b(x / \varepsilon, t)$ for all $x \in \Omega$.

To fix ideas, notice that the reaction rate $\eta$ may take the form

$\eta(\alpha, \beta)=\left\{\begin{array}{l}k_{3}^{\varepsilon}(x) \alpha^{p}(\bar{c}-\beta)^{q}, \quad \text { if } \alpha \geq 0, \beta \geq 0 \\ 0, \quad \text { otherwise, }\end{array}\right.$

where $\bar{c}$ is a known constant and $p \geq 1$ and $q \geq 1$ are partial reaction orders.

Since it is composed of mass-balance equations for some of the most relevant species participating in the corrosion process, the model (5)-(9) is rather standard. The reader is referred to [7,9] for more details on the engineering problem. Note that the structure of the power law (10) describing the reaction rate appears to be new in the context of sulfatation reactions. On the other hand, it is not at all clear how important is the precise structure of $\eta$ especially if one considers this process in the fast-reaction regime (i.e., for a fixed value of $\varepsilon$, let $k_{3}^{\varepsilon} \rightarrow \infty$ ). It is worth noting that this micro-model can be easily extended by allowing for ionic transport and the reaction of sulfate ions with the aluminate phases in concrete. Furthermore, we expect that more detailed modeling of the moisture behavior may replace the linear diffusion equation (8) with a porous media-type equation for the evolution of $u_{4}^{\varepsilon}$. A much more difficult step is to model the reaction-induced deformation of the microstructure and to account for the simultaneous space- and time-evolution of the active parts of the perforations.

\section{Nondimensionalization}

We introduce the characteristic length $L$ for the space variable such that $x=L \tilde{x}$, the time variable is scaled as $t=\tau s$, and for the concentrations we use $u_{i}^{\varepsilon}=u_{\text {ref }}^{i} v_{i}^{\varepsilon}$, where ${ }^{5} u_{\text {ref }}^{i}=\left\|u_{i}^{\varepsilon}\right\|_{\infty}$ for all $i \in\{1,2,3,4,5\} k_{j}$ are scaled

$\overline{5 L^{\infty} \text {-bounds }}$ on concentrations and the existence of positive weak solutions to the micro-model are shown in [31]. 
as $k_{j}^{\varepsilon}=k_{j}^{*} \tilde{k}_{j}^{\varepsilon}$, where $k_{j}^{*}=\left\|k_{j}^{\varepsilon}\right\|_{\infty}$ for all $j \in\{1,2,3\}$ and $d_{i}:=d_{\text {ref }}^{i} \tilde{d}_{i}$ for all $i \in\{1,2,3,4\}$. We make use of two mass-transfer Biot numbers ${ }^{6}$ for the two spatial scales in question: micro and macro. Our first Biot number is defined by

$\mathrm{Bi}^{m}:=\frac{b_{\text {ref }}^{m} L}{\mathfrak{D}}$,

where $b_{\text {ref }}^{m}$ is a reference reaction rate acting at the water solid interface within the microstructure and $\mathfrak{D}$ is a reference diffusion coefficient. Our second Biot number is defined by

$\mathrm{Bi}^{M}:=\frac{b_{\text {ref }}^{M} L}{\mathfrak{D}}$,

where $b_{\text {ref }}^{M}$ is a reference reaction rate at the water-solid interface at the macro level. The connection between the two Biot numbers is given by

$\mathrm{Bi}^{m}=\varepsilon \mathrm{Bi}^{M}$.

In some sense, relation (13) defines our small scaling parameter $\varepsilon$ with respect to which we wish to homogenize. Furthermore, we introduce two other dimensionless numbers:

$\beta_{i}:=\frac{u_{\mathrm{ref}}^{i}}{u_{\mathrm{ref}}^{1}}$ and $\gamma_{i}:=\frac{d_{\mathrm{ref}}^{i}}{d_{\mathrm{ref}}^{3}}$

$\beta_{i}$ represents the ratio of the maximum concentration of the $i$ th species to the maximum $\mathrm{H}_{2} \mathrm{SO}_{4}$ concentration, while $\gamma_{i}$ denotes the ratio of the characteristic time of the $i$ th diffusive aqueous species to the characteristic diffusion time of $\mathrm{H}_{2} \mathrm{~S}(g)$.

In terms of the newly introduced quantities, the mass-balance equation for $\mathrm{H}_{2} \mathrm{SO}_{4}$ takes the form

$\frac{u_{\mathrm{ref}}^{1}}{\tau} \partial_{s} v_{1}^{\varepsilon}+\frac{u_{\mathrm{ref}}^{1} d_{\mathrm{ref}}^{1}}{L^{2}} \operatorname{div}\left(-\tilde{d}_{1} \nabla v_{1}^{\varepsilon}\right)=-k_{1}^{*} u_{\mathrm{ref}}^{1} \tilde{k}_{1}^{\varepsilon} v_{1}^{\varepsilon}+k_{2}^{*} u_{\mathrm{ref}}^{2} \tilde{k}_{2}^{\varepsilon} v_{2}^{\varepsilon}$,

and hence,

$\beta_{1} \partial_{s} v_{1}^{\varepsilon}+\frac{\beta_{1} d_{\mathrm{ref}}^{1} \tau}{L^{2}} \operatorname{div}\left(-\tilde{d}_{1} \nabla v_{1}^{\varepsilon}\right)=-\frac{k_{1}^{*} u_{\mathrm{ref}}^{1} \tau}{u_{\mathrm{ref}}^{1}} \tilde{k}_{1}^{\varepsilon} v_{1}^{\varepsilon}+\frac{k_{2}^{*} u_{\mathrm{ref}}^{2} \tau}{u_{\mathrm{ref}}^{1}} \tilde{k}_{2}^{\varepsilon} v_{2}^{\varepsilon}$.

As reference time, we choose the characteristic time scale of the fastest species (here: $\mathrm{H}_{2} \mathrm{~S}(g)$ ), that is $\tau:=\tau_{\text {diff }}=$ $\frac{L^{2}}{d_{\text {ref }}^{3}}$. We get

$\beta_{1} \partial_{s} v_{1}^{\varepsilon}+\beta_{1} \gamma_{1} \operatorname{div}\left(-\tilde{d}_{1} \nabla v_{1}^{\varepsilon}\right)=-\frac{\eta_{\mathrm{ref}}^{1} \tau}{u_{\mathrm{ref}}^{1}} \tilde{k}_{1}^{\varepsilon} v_{1}^{\varepsilon}+\frac{\eta_{\mathrm{ref}}^{2} \tau}{u_{\mathrm{ref}}^{1}} \tilde{k}_{2}^{\varepsilon} v_{2}^{\varepsilon}$.

Let us denote by $\tau_{\text {reac }}^{j}:=\frac{u_{\text {ref }}^{1}}{\eta_{\text {ref }}^{j}}$ the characteristic time scale of the $j$ th reaction, where the quantity $\eta_{\text {ref }}^{j}$ is a reference reaction rate for the corresponding chemical reaction. With this new notation in hand, we obtain

$\beta_{1} \partial_{s} v_{1}^{\varepsilon}+\beta_{1} \gamma_{1} \operatorname{div}\left(-\tilde{d}_{1} \nabla v_{1}^{\varepsilon}\right)=-\Phi_{1}^{2} \tilde{k}_{1}^{\varepsilon} v_{1}^{\varepsilon}+\Phi_{2}^{2} \tilde{k}_{2}^{\varepsilon} v_{2}^{\varepsilon}$,

where $\Phi_{j}^{2}, j \in\{1,2,3\}$ are Thiele-like moduli. The $j$ th Thiele modulus $\Phi_{j}^{2}$ compares the characteristic time of the diffusion of the fastest species and the characteristic time of the $j$ th chemical reaction. It is defined as

$\Phi_{j}^{2}:=\frac{\tau_{\text {diff }}}{\tau_{\text {reac }}^{j}}$ for all $j \in\{1,2,3\}$.

For the boundary condition involving a surface reaction, we obtain

$\left.\tilde{n}_{\varepsilon} \cdot\left(-\tilde{d}_{1} \nabla v_{1}^{\varepsilon}\right)\right)=-\frac{\tau_{\text {diff }}}{\gamma_{1} L \tau_{\text {reac }}^{3}} \tilde{\eta}\left(v_{1}^{\varepsilon}, v_{5}^{\varepsilon}\right)$,

and therefore,

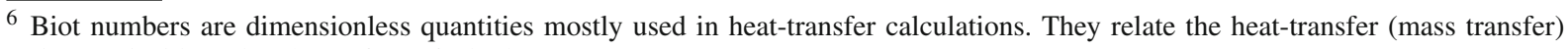
resistance inside and at the surface of a body. 
$\left.\tilde{n}_{\varepsilon} \cdot\left(-\tilde{d}_{1} \nabla v_{1}^{\varepsilon}\right)\right)=-\varepsilon \frac{\Phi_{3}^{2}}{\gamma_{1}} \tilde{\eta}\left(v_{1}^{\varepsilon}, v_{5}^{\varepsilon}\right)$.

Note that the quantity $\varepsilon \Phi_{3}^{2}$ plays the role of a Thiele modulus for a surface reaction, while $\Phi_{1}^{2}$ and $\Phi_{2}^{2}$ are Thiele moduli for volume reactions. Similarly, the mass-balance equation for the species $\mathrm{H}_{2} \mathrm{~S}(a q)$ becomes

$\beta_{2} \partial_{s} v_{2}^{\varepsilon}+\beta_{2} \gamma_{2} \operatorname{div}\left(-\tilde{d}_{2} \nabla v_{2}^{\varepsilon}\right)=\Phi_{1}^{2} \tilde{k}_{1} v_{1}^{\varepsilon}-\Phi_{2}^{2} \tilde{k}_{2} v_{2}^{\varepsilon}$.

The boundary condition at the air-water interface becomes

$\left.\tilde{n}_{\varepsilon} \cdot\left(-\tilde{d}_{2} \nabla v_{2}^{\varepsilon}\right)\right)=\varepsilon \mathrm{Bi}^{M}\left(\frac{a^{\varepsilon} \beta_{3}}{b^{\varepsilon} \beta_{2}} v_{3}^{\varepsilon}-v_{2}^{\varepsilon}\right)$.

The mass-balance equation for $\mathrm{H}_{2} \mathrm{~S}(\mathrm{~g})$ is

$\beta_{3} \partial_{s} v_{3}^{\varepsilon}+\beta_{3} \operatorname{div}\left(-\tilde{d}_{3} \nabla v_{3}^{\varepsilon}\right)=0$,

while the boundary condition at the air-water interface reads:

$\left.\tilde{n}_{\varepsilon} \cdot\left(-\tilde{d}_{3} \nabla v_{3}^{\varepsilon}\right)\right)=-\varepsilon \mathrm{Bi}^{M}\left(\frac{a^{\varepsilon}}{b^{\varepsilon}} v_{3}^{\varepsilon}-\frac{\beta_{2}}{\beta_{3}} v_{2}^{\varepsilon}\right)$.

Finally, the mass-balance equation for moisture is

$\beta_{4} \partial_{s} v_{4}^{\varepsilon}+\beta_{4} \gamma_{4} \operatorname{div}\left(-\tilde{d}_{4} \nabla v_{4}^{\varepsilon}\right)=\Phi_{1}^{2} \tilde{k}_{1} v_{1}^{\varepsilon}$

and the ODE for gypsum becomes

$\beta_{5} \partial_{s} v_{5}^{\varepsilon}=\Phi_{3}^{2} \tilde{\eta}\left(v_{1}^{\varepsilon}, v_{5}^{\varepsilon}\right)$.

To simplify the notation, we drop all the tildes and keep the meaning of the unknowns and operators as mentioned in this section.

\section{Formal homogenization procedure}

Homogenization is a generic term which refers to finding effective model equations and coefficients, i.e., objects independent of $\varepsilon$. For our problem, the homogenization procedure will provide us with an approximate macroscopic model (that we refer to as macro-model) defined for a uniform medium, where the original microstructure and phase separation (water, air, and solid) can no longer be seen. The hope is that the solutions to the macro-model are sufficiently close ${ }^{7}$ to the solutions of the micro-model as $\varepsilon$ goes to zero.

In this section, we study the asymptotic behaviour of the solutions to the micro-model as $\varepsilon \rightarrow 0$ for two parameter regimes reflecting two different types of diffusive-like transport of chemical species in concrete: "uniform" diffusion (see Sect. 4.1) and "structured" diffusion (Sect. 4.2).

\subsection{Case 1: $d_{i}^{\varepsilon}=\mathcal{O}(1)$ for all $i \in\{1,2,3,4\}$}

We consider that the diffusion speed is comparable for all concentrations, i.e., the diffusion coefficients $d_{i}^{\varepsilon}$ are of order of $\mathcal{O}(1)$ w.r.t. $\varepsilon$ for all $i \in\{1,2,3,4\}$. We assume that the solutions $v_{i}^{\varepsilon}(x, t), i \in\{1,2,3,4,5\}$ of the micro-model admit the following asymptotic expansion

$v_{i}^{\varepsilon}(x, t)=v_{i 0}(x, y, t)+\varepsilon v_{i 1}(x, y, t)+\varepsilon^{2} v_{i 2}(x, y, t)+\cdots$,

where $y=x / \varepsilon$ and the functions $v_{i m}(x, y, t), m=1,2,3, \cdots$, are $Y$-periodic in $y$.

\footnotetext{
7 The status of being "close" needs rigorous concepts (and proofs) that will be discussed in a forthcoming paper.
} 
If we define (compare [14,16], e.g.)

$\Psi_{\varepsilon}(x, t):=\Psi\left(x, \frac{x}{\varepsilon}, t\right)$,

then

$\frac{\partial \Psi_{\varepsilon}}{\partial x_{i}}=\frac{\partial \Psi}{\partial x_{i}}\left(x, \frac{x}{\varepsilon}\right)+\frac{1}{\varepsilon} \frac{\partial \Psi}{\partial y_{i}}\left(x, \frac{x}{\varepsilon}\right)$.

We investigate the asymptotic behavior of the solution $v_{1}^{\varepsilon}(x, t)$ as $\varepsilon \rightarrow 0$ of the following problem posed in the domain $Y_{\varepsilon}^{w}$

$\beta_{1} \partial_{s} v_{1}^{\varepsilon}+\beta_{1} \gamma_{1} \operatorname{div}\left(-d_{1} \nabla v_{1}^{\varepsilon}\right)=-\Phi_{1}^{2} k_{1}^{\varepsilon} v_{1}^{\varepsilon}+\Phi_{2}^{2} k_{2}^{\varepsilon} v_{2}^{\varepsilon}$ in $Y_{\varepsilon}^{w}$,

$\left.n_{\varepsilon} \cdot\left(-d_{1} \nabla v_{1}^{\varepsilon}\right)\right)=-\varepsilon \frac{\Phi_{3}^{2}}{\gamma_{1}} \eta\left(v_{1}^{\varepsilon}, v_{5}^{\varepsilon}\right)$ on $\Gamma_{\varepsilon}^{s w}$,

$\left.n_{\varepsilon} \cdot\left(-d_{1} \nabla v_{1}^{\varepsilon}\right)\right)=0$ on $\Gamma_{\varepsilon}^{w a}$.

Using now the asymptotic expansion of the solution $v_{1}^{\varepsilon}(x, t)$ in (30) and equating the terms with the same powers of $\varepsilon$, we obtain:

$\left\{\begin{array}{l}A_{0} v_{10}=0 \text { in } Y_{\varepsilon}^{w} \\ v_{10} \quad Y-\text { periodic in } y\end{array}\right.$

where the operator $A_{0}$ is given by

$A_{0}:=-\sum_{i, j=1}^{3} \frac{\partial}{\partial y_{i}}\left(d_{1}^{i j} \frac{\partial}{\partial y_{j}}\right)$.

As next step, we get

$\left\{\begin{array}{l}A_{0} v_{11}=-A_{1} v_{10} \text { in } Y_{\varepsilon}^{w}, \\ v_{11} \quad Y-\text { periodic in } y \\ \left(d_{1} \nabla_{y} v_{11}, \tilde{n}\right)=-\left(d_{1} \nabla_{x} v_{10}, \tilde{n}\right),\end{array}\right.$

where

$A_{1}:=-\sum_{i, j=1}^{3} \frac{\partial}{\partial x_{i}}\left(d_{1}^{i j} \frac{\partial}{\partial y_{j}}\right)-\sum_{i, j=1}^{3} \frac{\partial}{\partial y_{i}}\left(d_{1}^{i j} \frac{\partial}{\partial x_{j}}\right)$.

Furthermore, it holds that

$$
\begin{aligned}
\beta_{1} \gamma_{1} A_{0} v_{12}= & -\beta_{1} \gamma_{1} A_{1} v_{11}-\beta_{1} \gamma_{1} A_{2} v_{10}-\beta_{1} \partial_{s} v_{10}-\Phi_{1}^{2} k_{1}(y) v_{10}+\Phi_{2}^{2} k_{2}(y) v_{20} \text { in } Y_{\varepsilon}^{w}, \\
& v_{12} \quad Y-\text { periodic in } y, \\
\left(d_{1} \nabla_{y} v_{12}, \tilde{n}\right)= & -\left(d_{1} \nabla_{x} v_{11}, \tilde{n}\right)-\left(d_{1} \nabla_{x} v_{10}, n^{\prime}\right)-\left(d_{1} \nabla_{y} v_{11}, n^{\prime}\right)-\frac{\Phi_{3}^{2}}{\gamma_{1}} \eta\left(v_{10}, v_{50}\right) \text { on } \Gamma_{\varepsilon}^{s w}, \\
\left(d_{1} \nabla_{y} v_{12}, \tilde{n}\right)= & -\left(d_{1} \nabla_{x} v_{11}, \tilde{n}\right)-\left(d_{1} \nabla_{x} v_{10}, n^{\prime}\right)-\left(d_{1} \nabla_{y} v_{11}, n^{\prime}\right) \quad \text { on } \Gamma_{\varepsilon}^{w a},
\end{aligned}
$$

where

$A_{2}:=-\sum_{i, j=1}^{3} \frac{\partial}{\partial x_{i}}\left(d_{1}^{i j} \frac{\partial}{\partial x_{j}}\right)$.

From (31), we obtain that $v_{10}$ is independent of $y$. Since the elliptic equation for $v_{11}$ [with right-hand side defined in terms of $v_{10}$ ] is linear, its solution can be represented via

$v_{11}(x, y, t):=-\sum_{k=1}^{3} \chi^{k}(x, y, t) \frac{\partial v_{10}(x, t)}{\partial x_{k}}+v_{1}(x, t)$, 
where the functions $\chi^{k}(x, y, t)$ solve the cell problem(s) and are periodic w.r.t. $y$. In the rest of the paper, we do not point out anymore the dependence of $\chi^{k}$ on the parameter $t$. The exact expression of $v_{1}$ does not matter much at this stage. Using the expression of $v_{11}$, we obtain following cell problems in the standard manner:

$$
\begin{aligned}
& A_{0} \chi^{k}(x, y)=-\sum_{i=1}^{3} \frac{\partial}{\partial y_{i}} d_{1}^{i k}(y), k \in\{1,2,3\} \text { in } Y_{x}^{w}, \\
& \sum_{i, j, k=1}^{3} \frac{\partial v_{10}}{\partial x_{k}}\left[d_{1}^{i j} \frac{\partial \chi^{k}}{\partial y_{j}} \tilde{n}_{i}-d_{1}^{j k} \tilde{n}_{j}\right]=0, \quad \text { on } \Gamma_{x}^{s w}, \\
& \sum_{i, j, k=1}^{3} \frac{\partial v_{10}}{\partial x_{k}}\left[d_{1}^{i j} \frac{\partial \chi^{k}}{\partial y_{j}} \tilde{n}_{i}-d_{1}^{j k} \tilde{n}_{j}\right]=0, \quad \text { on } \Gamma_{x}^{w a} .
\end{aligned}
$$

In (36) the cell function $\chi^{k}$ inherits the $x$-dependence from the perforation, and hence, instead of a standard periodic cell $Y$, we now deal with a $x$-dependent family of cells $Y_{x}$.

Since the right-hand side of (36) integrated over $Y_{x}^{w}$ is zero, this problem has a unique solution. Note also that (32) leads to

$$
\begin{aligned}
\beta_{1} \gamma_{1} A_{0} v_{12}= & \beta_{1} \gamma_{1}\left[-\sum_{i, j, k=1}^{3} \frac{\partial v_{10}}{\partial x_{k}} \frac{\partial}{\partial y_{i}}\left(d_{1}^{i j} \frac{\partial \chi^{k}}{\partial x_{j}}\right)\right. \\
& -\sum_{i, j, k=1}^{3} \frac{\partial^{2} v_{10}}{\partial x_{j} \partial x_{k}} \frac{\partial}{\partial y_{i}}\left(d_{1}^{i j} \chi^{k}\right)+\sum_{i, j=1}^{3} \frac{\partial d_{1}^{i j}}{\partial y_{i}} \frac{\partial \tilde{v}_{1}}{\partial x_{j}}-\sum_{i, j, k=1}^{3} d_{1}^{i j} \frac{\partial^{2} \chi^{k}}{\partial x_{i} \partial y_{j}} \frac{\partial v_{10}}{\partial x_{k}}-\sum_{i, j, k=1}^{3} d_{1}^{i j} \frac{\partial \chi^{k}}{\partial y_{i}} \frac{\partial^{2} v_{10}}{\partial x_{k} \partial x_{i}} \\
& \left.+\sum_{i, k=1}^{3} d_{1}^{i k} \frac{\partial^{2} v_{10}}{\partial x_{k} \partial x_{i}}\right]-\beta_{1} \partial_{s} v_{10}-\Phi_{1}^{2} k_{1}(y) v_{10}+\Phi_{2}^{2} k_{2}(y) v_{20} .
\end{aligned}
$$

Moreover, we have

$$
\begin{aligned}
\beta_{1} \gamma_{1}\left(d_{1} \nabla_{y} v_{12}, \tilde{n}\right)= & \beta_{1} \gamma_{1}\left[\sum_{i, j, k=1}^{3} d_{1}^{i j} \frac{\partial v_{10}}{\partial x_{k}} \frac{\partial \chi^{k}}{\partial x_{i}} \tilde{n}_{j}+\sum_{i, j, k=1}^{3} d_{1}^{i j} \frac{\partial^{2} v_{10}}{\partial x_{j} \partial x_{k}} \chi^{k} \tilde{n}_{j}-\sum_{i, j=1}^{3} d_{1}^{i j} \frac{\partial v_{10}}{\partial x_{i}} n_{j}^{\prime}\right. \\
& \left.-\sum_{i, j=1}^{3} d_{1}^{i j} \frac{\partial \tilde{v}_{1}}{\partial x_{i}} \tilde{n}_{j}+\sum_{i, j, k=1}^{3} d_{1}^{i j} \frac{\partial \chi^{k}}{\partial x_{i}} \frac{\partial v_{10}}{\partial x_{k}} n_{j}^{\prime}-\frac{\Phi_{3}^{2}}{\gamma_{1}} \eta\left(v_{10}, v_{50}\right)\right] .
\end{aligned}
$$

Writing down the compatibility condition (see e.g. [15, Lemma 2.1]), we get

$$
\begin{aligned}
& \int_{Y_{x}^{w}}\left[\beta _ { 1 } \gamma _ { 1 } \left\{\sum_{i, j, k=1}^{3} \frac{\partial v_{10}}{\partial x_{k}} \frac{\partial}{\partial y_{i}}\left(d_{1}^{i j} \frac{\partial \chi^{k}}{\partial x_{j}}\right)+\sum_{i, j, k=1}^{3} \frac{\partial^{2} v_{10}}{\partial x_{j} \partial x_{k}} \frac{\partial}{\partial y_{i}}\left(d_{1}^{i j} \chi^{k}\right)\right.\right. \\
& \left.-\sum_{i, j=1}^{3} \frac{\partial d_{1}^{i j}}{\partial y_{i}} \frac{\partial \tilde{v}_{1}}{\partial x_{j}}+\sum_{i, j, k=1}^{3} d_{1}^{i j} \frac{\partial^{2} \chi^{k}}{\partial x_{i} \partial y_{j}} \frac{\partial v_{10}}{\partial x_{k}}+\sum_{i, j, k=1}^{3} d_{1}^{i j} \frac{\partial \chi^{k}}{\partial y_{i}} \frac{\partial^{2} v_{10}}{\partial x_{k} \partial x_{i}}-\sum_{i, j, k=1}^{3} d_{1}^{i j} \frac{\partial^{2} v_{10}}{\partial x_{j} \partial x_{i}}\right\} \\
& \left.+\beta_{1} \partial_{s} v_{10}+\Phi_{1}^{2} k_{1}(y) v_{10}-\Phi_{2}^{2} k_{2}(y) v_{20}\right] \mathrm{d} y=\beta_{1} \gamma_{1} \int_{\Gamma_{x}^{s w}}\left[\sum_{i, j, k=1}^{3} d_{1}^{i j} \frac{\partial v_{10}}{\partial x_{k}} \frac{\partial \chi^{k}}{\partial x_{i}} \tilde{n}_{j}\right. \\
& +\int_{\Gamma_{x}^{s w}}\left[\sum_{i, j, k=1}^{3} d_{1}^{i j} \frac{\partial^{2} v_{10}}{\partial x_{j} \partial x_{k}} \chi^{k} \tilde{n}_{j}-\sum_{i, j=1}^{3} d_{1}^{i j} \frac{\partial v_{10}}{\partial x_{i}} n_{j}^{\prime}\right] \mathrm{d} \sigma \\
& \left.-\int_{\Gamma_{x}^{s w}}\left[\sum_{i, j=1}^{3} d_{1}^{i j} \frac{\partial \tilde{v}_{1}}{\partial x_{i}} \tilde{n}_{j}+\sum_{i, j, k=1}^{3} d_{1}^{i j} \frac{\partial \chi^{k}}{\partial x_{i}} \frac{\partial v_{10}}{\partial x_{k}} n_{j}^{\prime}\right] \mathrm{d} \sigma_{y}-\int_{\Gamma_{x}^{s w}} \frac{\Phi_{3}^{2}}{\gamma_{1}} \eta\left(v_{10}, v_{50}\right)\right] \mathrm{d} \sigma_{y} .
\end{aligned}
$$


We apply Stokes' theorem to the terms involving $\tilde{n}_{j}$ and after straightforward calculations, obtain

$$
\begin{aligned}
& \beta_{1} \partial_{s} v_{10}+\Phi_{1}^{2} v_{10} \frac{1}{\left|Y_{x}^{w}\right|} \int_{Y_{x}^{w}} k_{1}(y) \mathrm{d} y-\Phi_{2}^{2} v_{20} \frac{1}{\left|Y_{x}^{w}\right|} \int_{Y_{x}^{w}} k_{2}(y) \mathrm{d} y-\beta_{1} \gamma_{1} \sum_{i, j, k=1}^{3} \frac{\partial^{2} v_{10}}{\partial x_{i} \partial x_{k}}\left\langle d_{1}^{i j} \frac{\partial \chi^{k}}{\partial y_{j}}-d_{1}^{i k}\right\rangle \\
& -\beta_{1} \gamma_{1} \sum_{i, j, k=1}^{3}\left\langle d_{1}^{i j} \frac{\partial^{2} \chi^{k}}{\partial x_{i} \partial y_{j}}\right) \frac{\partial v_{10}}{\partial x_{k}}=-\beta_{1} \gamma_{1} \sum_{i, j, k=1}^{3} \frac{\partial v_{10}}{\partial x_{k}} \frac{1}{\left|Y_{x}^{w}\right|} \int_{\Gamma_{x}^{s w}}\left(d_{1}^{k j} n_{j}^{\prime}-d_{1}^{i j} \frac{\partial \chi^{k}}{\partial y_{i} n_{j}^{\prime}}\right) \mathrm{d} \sigma_{y} \\
& -\frac{\beta_{1} \gamma_{1}}{\gamma_{1}} \Phi_{3}^{2} v_{10} \frac{1}{\left|Y_{x}^{w}\right|} \int_{\Gamma_{x}^{s w}} v_{50}(x, y, t) k_{3}(y) \mathrm{d} \sigma_{y},
\end{aligned}
$$

where $\langle f\rangle_{V}:=\frac{1}{|V|} \int_{V} f \mathrm{~d} x$ is for any $V$ a subset of either $Y_{x}^{a}$ or $Y_{x}^{w}$ and $|V|$ is the volume of $V$. The latter PDE can be rewritten as

$$
\begin{gathered}
\beta_{1} \partial_{s} v_{10}-\beta_{1} \gamma_{1} \sum_{i, j, k=1}^{3} \frac{\partial}{\partial x_{i}}\left(\left\langle d_{1}^{i j} \frac{\partial \chi^{k}}{\partial y_{j}}-d_{1}^{i k}\right\rangle \frac{\partial v_{10}}{\partial x_{k}}\right)+\Phi_{1}^{2} v_{10} K_{1}-\Phi_{2}^{2} v_{20} K_{2} \\
=-\beta_{1} \gamma_{1} \sum_{k=1}^{3} \frac{\partial v_{10}}{\partial x_{k}} U_{k}-\beta_{1} \Phi_{3}^{2} v_{10} K_{3} \text { in } \Omega,
\end{gathered}
$$

where

$$
\begin{aligned}
& K_{\ell}(x):=\frac{1}{\left|Y_{x}^{w}\right|} \int_{Y_{x}^{w}} k_{\ell}(y) \mathrm{d} y, \ell \in\{1,2\}, \\
& K_{3}(x):=\frac{1}{\left|Y_{x}^{w}\right|} \int_{\Gamma_{x}^{s w}} v_{50}(x, y, t) k_{3}(y) \mathrm{d} \sigma_{y},
\end{aligned}
$$

and

$U_{k}(x):=\frac{1}{\left|Y_{x}^{w}\right|} \sum_{i, j=1}^{3} \int_{\Gamma_{x}^{s w}}\left(d_{1}^{k j} n_{j}^{\prime}-d_{1}^{i j} \frac{\partial \chi^{k}}{\partial y_{i}} n_{j}^{\prime}\right) \mathrm{d} \sigma_{y}$.

The terms $U_{k}$ are new. They occur due to the assumed deviation from a uniformly periodic distribution of perforations.

Now we apply the same procedure to the next mass-balance equation. To do this, we consider the auxiliary cell problem

$$
\begin{aligned}
& A_{0} \chi^{k}(x, y)=-\sum_{i=1}^{3} \frac{\partial}{\partial y_{i}} d_{2}^{i k}(y), k \in\{1,2,3\} \text { in } Y_{x}^{w}, \\
& \sum_{i, j, k=1}^{3} \frac{\partial v_{10}}{\partial x_{k}}\left[d_{2}^{i j} \frac{\partial \chi^{k}}{\partial y_{j}} \tilde{n}_{i}-d_{2}^{j k} \tilde{n}_{j}\right]=0, \quad \text { on } \Gamma_{x}^{s w}, \\
& \sum_{i, j, k=1}^{3} \frac{\partial v_{10}}{\partial x_{k}}\left[d_{2}^{i j} \frac{\partial \chi^{k}}{\partial y_{j}} \tilde{n}_{i}-d_{2}^{j k} \tilde{n}_{j}\right]=0, \quad \text { on } \Gamma_{x}^{w a},
\end{aligned}
$$

whose solution is $\chi^{k}(x, y)$. We obtain the upscaled PDE:

$$
\begin{aligned}
& \beta_{2} \partial_{s} v_{20}-\Phi_{1}^{2} v_{10} k_{1}+\Phi_{2}^{2} v_{20} k_{2}-\beta_{2} \gamma_{2} \sum_{i, j, k=1}^{3} \frac{\partial}{\partial x_{i}}\left(\left\langle d_{2}^{i j} \frac{\partial \chi^{k}}{\partial y_{j}}-d_{2}^{i k}\right\rangle \frac{\partial v_{20}}{\partial x_{k}}\right) \\
& =-\beta_{2} \gamma_{2} \sum_{k=1}^{3} \frac{\partial v_{20}}{\partial x_{k}} U_{k}-\beta_{3} \mathrm{Bi}^{M} v_{30} C+\beta_{2} \mathrm{Bi}^{M} v_{20} B,
\end{aligned}
$$

holding in $\Omega$, where 


$$
\begin{aligned}
C(x) & :=\frac{1}{\left|Y_{x}^{w}\right|} \int_{\Gamma_{x}^{w a}} b(y) H(y) \mathrm{d} \sigma_{y}, \\
H(y) & :=\frac{a(y)}{b(y)}, \quad \text { with } y \in \Gamma_{x}^{w a}, \\
B(x) & :=\frac{1}{\left|Y_{x}^{w}\right|} \int_{\Gamma_{x}^{w a}} b(y) \mathrm{d} \sigma_{y}, \\
U_{k}(x) & :=\frac{1}{\left|Y_{x}^{w}\right|} \sum_{i, j=1}^{3} \int_{\Gamma_{x}^{w a}}\left(d_{1}^{k j} n_{j}^{\prime}-d_{1}^{i j} \frac{\partial \chi^{k}}{\partial y_{i}} n_{j}^{\prime}\right) \mathrm{d} \sigma_{y} .
\end{aligned}
$$

We now treat the mass-balance equation for $\mathrm{H}_{2} \mathrm{~S}(\mathrm{~g})$. The corresponding cell problems are given by

$$
\begin{aligned}
& A_{0} \chi^{k}(x, y)=-\sum_{i=1}^{3} \frac{\partial}{\partial y_{i}} d_{3}^{i k}(y), k=1,2,3 \text { in } Y_{x}^{a}, \\
& \sum_{j, k=1}^{3} \frac{\partial v_{30}}{\partial x_{k}}\left[\sum_{i=1}^{3} d_{3}^{i j} \frac{\partial \chi^{k}}{\partial y_{j}} \tilde{n}_{i}-d_{3}^{j k} \tilde{n}_{j}\right]=0 \text { on } \Gamma_{x}^{w a}, \\
& \sum_{j, k=1}^{3} \frac{\partial v_{30}}{\partial x_{k}}\left[\sum_{i=1}^{3} d_{3}^{i j} \frac{\partial \chi^{k}}{\partial y_{j}} \tilde{n}_{i}-d_{3}^{j k} \tilde{n}_{j}\right]=0 \text { on } \partial Y_{x}^{a}-\Gamma_{x}^{w a},
\end{aligned}
$$

while the macroscopic PDE is

$\partial_{s} v_{30}-\sum_{i, j, k=1}^{3} \frac{\partial}{\partial x_{i}}\left(\left\langle d_{3}^{i j} \frac{\partial \chi^{k}}{\partial y_{j}}-d_{3}^{i k}\right\rangle \frac{\partial v_{30}}{\partial x_{k}}\right)=-\sum_{k=1}^{3} \frac{\partial v_{30}}{\partial x_{k}} U_{k}+\beta_{3} \mathrm{Bi}^{M} v_{30} C-\beta_{2} \mathrm{Bi}^{M} v_{20} B$

in $\Omega$ with $v_{30}=v_{30}^{D}$ on $\Gamma^{D}$. Here we have

$$
\begin{aligned}
& C(x):=\frac{1}{\left|Y_{x}^{a}\right|} \int_{\Gamma_{x}^{w a}} b(y) H(y) \mathrm{d} \sigma_{y}, \\
& B(x):=\frac{1}{\left|Y_{x}^{a}\right|} \int_{\Gamma_{x}^{w a}} b(y) \mathrm{d} \sigma_{y} .
\end{aligned}
$$

The same procedure leads to

$\beta_{4} \partial_{s} v_{40}-\Phi_{1}^{2} v_{10} k_{1}-\beta_{4} \gamma_{4} \sum_{i, j, k=1}^{3} \frac{\partial}{\partial x_{i}}\left(\left\langle d_{4}^{i j} \frac{\partial \chi^{k}}{\partial y_{j}}-d_{4}^{i k}\right) \frac{\partial v_{40}}{\partial x_{k}}\right)=-\beta_{4} \gamma_{4} \sum_{k=1}^{3} \frac{\partial v_{40}}{\partial x_{k}} U_{k}$,

in $\Omega$ with $v_{40}=0$, on $\Gamma$.

Interestingly, the case of the ODE for gypsum

$\partial_{s} v_{5}^{\varepsilon}=\Phi_{3}^{2} \eta\left(v_{1}^{\varepsilon}, v_{5}^{\varepsilon}\right) \quad$ on $\Gamma_{\varepsilon}^{s w}, s \in S$,

$v_{5}^{\varepsilon}(x, 0)=v_{5}^{\varepsilon}(x)$,

seems to be more problematic. Let us first use the same homogenization ansatz as before and employ $\tilde{\eta}\left(v_{1}^{\varepsilon}, v_{5}^{\varepsilon}\right)=\eta_{0}^{A}\left(v_{10}(x, t), v_{50}(x, y, t)\right)+\mathcal{O}(\varepsilon)$.

We obtain

$$
\begin{aligned}
& \partial_{s} v_{50}(x, y, t)=\Phi_{3}^{2} \eta_{0}^{A}\left(v_{10}(x, t), v_{50}(x, y, t)\right) \quad \text { with } y \in \Gamma_{x}^{s w}, \\
& v_{50}(x, y, 0)=v_{50}(x, y),
\end{aligned}
$$


where $v_{50}(x, y, t)$ is periodic w.r.t $y$. Notice that we can not obtain an expression for $v_{50}(x, y, t)$ that is independent of $y$ ! On the other hand, if we make another ansatz for $v_{5}^{\varepsilon}$, say

$v_{5}^{\varepsilon}(x, t)=v_{50}(x, t)+\varepsilon v_{51}(x, y, t)+\varepsilon^{2} v_{52}(x, y, t)+\ldots$,

then

$\tilde{\eta}\left(v_{1}^{\varepsilon}, v_{5}^{\varepsilon}\right)=\eta_{0}^{B}\left(v_{10}(x, t), v_{50}(x, t)\right)+\mathcal{O}(\varepsilon)$

and we obtain an averaged ODE independent of $y$ as given via

$\partial_{s} v_{50}(x, t)=\Phi_{3}^{2} \eta_{0}^{B}\left(v_{10}(x, t), v_{50}(x, t)\right)$.

The advantage of the second choice is that it leads to the averaged reaction constant $\bar{k}_{3}=\frac{1}{\left|\Gamma_{x}^{s w}\right|} \int_{\Gamma_{x}^{s w}} k_{3}(y) \mathrm{d} y$, which is, in practice, much nicer than (56). Summarizing: we have to choose between (56) and (59), but which of the two averaged ODEs is the right one? Does the correctness of the answer to this question depend on the choice of the initial datum for $v_{50}$ ? We will address these issues ${ }^{8}$ in a forthcoming paper where we justify rigorously the asymptotic behavior indicated here.

4.2 Case 2: $d_{3}^{\varepsilon}=\mathcal{O}(1)$ and $d_{i}^{\varepsilon}=\mathcal{O}\left(\varepsilon^{2}\right)$ for all $i \in\{1,2,4\}$

In this section, we take into account the fact that the diffusion of $\mathrm{H}_{2} \mathrm{~S}$ is much faster within the air-part of the pores than within the pore water. Particularly, we assume that $d_{3}^{\varepsilon}$ is of order of $\mathcal{O}(1)$, while $d_{i}^{\varepsilon}=\mathcal{O}\left(\varepsilon^{2}\right)$ for all $i \in\{1,2,4\}$. We expect from the literature that the latter assumption will lead to a two-scale model for which the micro- and macro-structure need to be resolved simultaneously; see e.g. [25, 27,29].

Assume the initial data to be given by $v_{i}^{\varepsilon}(x, 0)=v_{i}^{0}\left(x, \frac{x}{\varepsilon}\right), \quad i \in\{1,2,3,4,5\}$ with functions $v_{i}^{0}: \Omega \times Y \times S \rightarrow \mathbb{R}$ being $Y$-periodic with respect to the second variable $y \in Y$. Assume also that $d_{i}^{\varepsilon}=\varepsilon^{2} d_{i}^{0}$, for $i \in\{1,2,4\}$ and $d_{3}^{\varepsilon}=d_{3}^{0}$. We then employ the same homogenization ansatz

$v_{i}^{\varepsilon}(x, t)=w_{i 0}(x, y, t)+\varepsilon w_{i 1}(x, y, t)+\varepsilon^{2} w_{i 2}(x, y, t)+\cdots$

for all $i \in\{1,2,3,4,5\}$. Using the same strategy as in Sect. 4.1, we obtain

$\beta_{1} \partial_{s} w_{10}(x, y, t)-\beta_{1} \gamma_{1} \nabla_{y} \cdot\left(d_{1}^{0} \nabla_{y} w_{10}(x, y, t)\right)=-k_{1}(y) w_{10}(x, y, t)+k_{2}(y) w_{20}(x, y, t)$

in $\Omega \times Y_{x}^{w} \times S$. The boundary conditions become

$\tilde{n}(x, y) \cdot\left(-d_{1}^{0} \nabla_{y} w_{10}(x, y, t)\right)=0$ on $\Omega \times \Gamma_{x}^{w a} \times S$,

$\tilde{n}(x, y) \cdot\left(-d_{1}^{0} \nabla_{y} w_{10}(x, y, t)\right)=-\frac{\Phi_{3}^{2}}{\gamma_{3}} k_{3}(y) w_{10}(x, y, t) w_{50}(x, y, t)$

on $\Omega \times \Gamma_{x}^{s w} \times S$.

Similarly,

$\beta_{2} \partial_{s} w_{20}(x, y, t)-\beta_{2} \gamma_{2} \nabla_{y} \cdot\left(d_{2}^{0} \nabla_{y} w_{20}(x, y, t)\right)=k_{1}(y) w_{10}(x, y, t)-k_{2}(y) w_{20}(x, y, t)$,

in $\Omega \times Y_{x}^{w} \times S$ while the boundary conditions take the form

$\tilde{n}(x, y) \cdot\left(-d_{2}^{0} \nabla_{y} w_{20}(x, y, t)\right)=0$ on $\Omega \times \Gamma_{x}^{s w} \times S$,

$\tilde{n}(x, y) \cdot\left(-d_{2}^{0} \nabla_{y} w_{20}(x, y, t)\right)=\mathrm{Bi}^{M} b(y) \times\left[\frac{\beta_{3}}{\beta_{2}} H(y) w_{30}(x, t)-w_{20}(x, y, t)\right]$ on $\Omega \times \Gamma_{x}^{w a} \times S$.

\footnotetext{
8 To some extent we anticipate here the answer to the latter question: trusting [20], we can prove relation (56) rigorously via a two-scale convergence approach. However, we will show later that, under some additional conditions on the initial data, that (56) reduces to (59).
} 
Since we consider $d_{3}^{\varepsilon}=d_{3}^{0}$, we obtain the same macroscopic PDE as in Case 1:

$$
\begin{aligned}
& \partial_{s} w_{30}(x, t)-\sum_{i, j, k=1}^{3} \frac{\partial}{\partial x_{i}}\left(\left\langle d_{3}^{i j} \frac{\partial \chi^{k}}{\partial y_{j}}-d_{3}^{i k}\right\rangle \frac{\partial w_{30}(x, t)}{\partial x_{k}}\right) \\
& =-\sum_{k=1}^{3} \frac{\partial w_{30}(x, t)}{\partial x_{k}} U_{k}+\frac{\beta_{3} \mathrm{Bi}^{M} w_{30}(x, t)}{\left|Y_{x}^{w}\right|} \int_{\Gamma_{x}^{w a}} b(y) H(y) \mathrm{d} \sigma_{y}-\frac{\beta_{2} \mathrm{Bi}^{M}}{\left|Y_{x}^{w}\right|} \int_{\Gamma_{x}^{w a}} b(y) w_{20}(x, y, t) \mathrm{d} \sigma_{y}
\end{aligned}
$$

in $\Omega$ and

$w_{30}(x, t)=w_{30}^{D}(x, t)$ on $\Gamma^{D}$,

where

$U_{k}(x):=\frac{1}{\left|Y_{x}^{w}\right|} \sum_{i, j=1}^{3} \int_{\Gamma_{x}^{w a}}\left(d_{1}^{k j} n_{j}^{\prime}-d_{1}^{i j} \frac{\partial \chi^{k}}{\partial y_{i}} n_{j}^{\prime}\right) \mathrm{d} \sigma_{y}$.

Next, we have

$\beta_{4} \partial_{s} w_{40}(x, y, t)-\beta_{4} \gamma_{4} \nabla_{y} .\left(d_{4}^{0} \nabla_{y} w_{40}(x, y, t)\right)=k_{1}(y) w_{10}(x, y, t)$

on $\Omega \times Y^{w} \times S$, while the boundary conditions are now given by

$$
\begin{array}{ll}
\tilde{n}(x, y) \cdot\left(-d_{4}^{0} \nabla_{y} w_{40}(x, y, t)\right)=0 & \text { on } \Omega \times \Gamma_{x}^{w a} \times S, \\
\tilde{n}(x, y) \cdot\left(-d_{4}^{0} \nabla_{y} w_{40}(x, y, t)\right)=0 & \text { on } \Omega \times \Gamma_{x}^{s w} \times S .
\end{array}
$$

The ODE modeling gypsum growth takes the form

$\beta_{5} \partial_{s} w_{50}(x, y, t)=-\Phi_{3}^{2} \eta\left(w_{10}(x, y, t) w_{50}(x, y, t)\right)$

on $\Omega \times \Gamma_{x}^{s w} \times S$.

\section{Conclusion}

We have discussed a reaction-diffusion system modeling sulfate corrosion of sewer pipes, where the porous material (concrete) was assumed to have a locally periodic perforated structure. Having in mind this special choice of microstructures, we used asymptotic homogenization techniques to obtain upscaled reaction-diffusion models (together with explicit formulae for the effective transport and reaction coefficients) for two relevant parameter regimes: (a) all diffusion coefficients are of order of $\mathcal{O}(1)$ and (b) all diffusion coefficients are of order of $\mathcal{O}\left(\varepsilon^{2}\right)$ except the one for $\mathrm{H}_{2} \mathrm{~S}(\mathrm{~g})$ which is of order of $\mathcal{O}(1)$. In case $(a)$, we obtained a set of macroscopic equations, while in case $(b)$ we are led to a two-(spatial)scale reaction-diffusion system. In this context, the main remaining open issue is to justify rigorously these asymptotic behaviors for uniformly periodic case. We will address closely related aspects in [31].

As future plans, we wish to perform extensive simulations for the two-scale model (61)-(72) for the case of a fixed geometry. This should help understanding the long-time behavior of the concentrations for the case of matched micro-macro transmission conditions starting from regularized ones (with a large Biot number). On the other hand, we would like to extend the two-scale sulfatation model when (69) is replaced by a strongly nonlinear pde describing the wetness of concrete.

Acknowledgments We acknowledge fruitful multiscale-related discussions with Tycho van Noorden. Also, we would like to thank Prof. Luisa Mascarenhas for providing Ref. [21].

Open Access This article is distributed under the terms of the Creative Commons Attribution Noncommercial License which permits any noncommercial use, distribution, and reproduction in any medium, provided the original author(s) and source are credited. 


\section{References}

1. Hewlett PC (1998) Lea's Chemistry of cement and concrete. Elsevier Butterworth-Heinemenn Linacre House, Oxford 327-328

2. Beddoe RE, Dorner HW (2005) Modelling acid attack on concrete: part 1. The essential mechanisms. Cem Concr Res 35:2333-2339

3. Beddoe RE (2009) Modelling the evolution of damage to concrete by acid attack. In: Franke L (ed) Simulation of time dependent degradation of porous materials: final report on priority program 1122. Cuvillier Verlag, Göttingen, pp 275-293

4. Song HW, Lim HJ, Saraswathy V, Kim TH (2007) A micro-mechanics based corrosion model for predicting the service life of reinforced concrete structures. Int J Electr Sci 2:341-354

5. Müllauer W, Beddoe RE, Heinz D (2009) Sulfate attack on concrete-solution concentration and phase stability. In: Concrete in aggressive aqueous environments, performance, testing and modeling. Toulouse, France, pp 18-27

6. Marchand J, Samson E, Maltais Y, Lee RJ, Sahu S (2002) Predicting the performance of concrete structures exposed to chemically aggressive environment-field validation. Mater Struct 35:623-631

7. Tixier R, Mobasher B, Asce M (2003) Modeling of damage in cement-based materials subjected to external sulfate attack. I: formulation. J Mater Civil Eng 15:305-313

8. Taylor HFW (1990) Cement chemistry. Academic Press, London

9. Böhm M, Jahani F, Devinny J, Rosen G (1998) A moving-boundary system modeling corrosion of sewer pipes. Appl Math Comput 92:247-269

10. Jahani F, Devinny J, Mansfeld F, Rosen G, Sun Z, Wang C (2001) Investigations of sulfuric acid corrosion of the concrete, I: modeling and chemical observations. J Environ Eng 127(7):572-579

11. Jahani F, Devinny J, Mansfeld F, Rosen G, Sun Z, Wang C (2001) Investigations of sulfuric acid corrosion of the concrete, II: electrochemical and visual observations. J Environ Eng 127(7):580-584

12. Agreba-Driolett D, Diele F, Natalini R (2004) A mathematical model for the $\mathrm{SO}_{2}$ aggression to calcium carbonate stones: numerical approximation and asymptotic analysis. SIAM J Appl Math 64(5):1636-1667

13. Auriault J-L (1991) Heterogeneous medium: is an equivalent macroscopic description possible? Int J Eng Sci 29(7):785-795

14. Bensoussan A, Lions JL, Papanicolau G (1978) Asymptotic analysis for periodic structures. North-Holland, Amsterdam

15. Persson LE, Persson L, Svanstedt N, Wyller J (1993) The homogenization method. Chartwell Bratt, Lund

16. Cioranescu D, Donato P (1999) An introduction to homogenization. Oxford University Press, New York

17. van Duijn C, Mikelic A, Pop IS, Rosier C (2008) Effective dispersion equations for reactive flows with dominant Peclet and Damköhler numbers. In: Marin GB, West D, Yablonsky GS (eds) Mathematics in chemical kinetics and engineering. Academic Press, New York, pp 1-45

18. Chechkin GA, Chechkina TP (2004) On homogenization of problems in domains of the "infusorium" type. J Math Sci 120(3):386407

19. Hornung U, Jäger W (1991) Diffusion, convection, adsorption, and reaction of chemicals in porous media. J Diff Equ 92:199-225

20. Marciniak-Czochra A, Ptashnyk M (2008) Derivation of a macroscopic receptor-based model using homogenization techniques. SIAM J Math Anal 40(1):215-237

21. Mascarenhas ML (1995) Homogenization problems in locally periodic perforrated domains. In: Procedings of the international conference in asymptotic methods for elastic structures, Berlin, pp 141-149

22. Belyaev AG, Pyatnitskii AL, Chechkin GA (1998) Asymptotic behaviour of a solution to a boundary value problem in a perforated domain with oscillating boundary. Siber Math J 39(4):621-644

23. Chechkin G, Pianitskii AL (1999) Homogenization of boundary-value problem in a locally periodic perforated domain. Appl Anal 71(1):215-235

24. Chechkin GA, Piatnitskii AL, Shamaev AS (2007) Homogenization. Methods and applications, vol 234 of translations of mathematical monographs. AMS, Providence

25. Eck C (2004) Homogenization of a phase field model for binary mixtures. Multiscale Model Simul 3(1):1-27

26. Meier SA (2008) Two-scale models for reactive transport and evolving microstructure. PhD thesis, University of Bremen, Germany

27. Meier SA, Muntean A (2009) A two-scale reaction-diffusion system with micro-cell reaction concentrated on a free boundary. Comp Rendus Mécan 336(6):481-486

28. Meier SA, Peter MA, Muntean A, Böhm M, Kropp J (2007) A two-scale approach to concrete carbonation. In: Proceedings of the first international RILEM workshop on integral service life modeling of concrete structures, Guimaraes, Portugal, pp 3-10

29. Hornung U, Jäger W, Mikelic A (1994) Reactive transport through an array of cells with semi-permeable membranes. RAIRO Model Math Anal Numer 28(1):59-94

30. van Noorden T (2009) Crystal precipitation and dissolution in a porous medium: effective equations and numerical experiments. Multiscale Model Simul 7(3):1220-1236

31. Fatima T, Muntean A (2010) Well-posedness and two-scale convergence for a pore model describing sulfate corrosion (in preparation) 